\title{
Review Article \\ Concepts and Treatment Approaches in Nonalcoholic Fatty Liver Disease
}

\author{
Dina L. Halegoua-De Marzio and Jonathan M. Fenkel \\ Division of Gastroenterology and Hepatology, Department of Medicine, Thomas Jefferson University Hospital, \\ 132 South 10th Street, Philadelphia, PA 19107, USA
}

Correspondence should be addressed to Dina L. Halegoua-De Marzio; dina.halegoua@gmail.com

Received 20 August 2014; Accepted 14 November 2014; Published 30 November 2014

Academic Editor: Junyong Park

Copyright (C) 2014 D. L. Halegoua-De Marzio and J. M. Fenkel. This is an open access article distributed under the Creative Commons Attribution License, which permits unrestricted use, distribution, and reproduction in any medium, provided the original work is properly cited.

\begin{abstract}
Nonalcoholic fatty liver disease (NAFLD) affects up to $30 \%$ of adults and is the most common liver disease in Western nations. NAFLD is associated with central adiposity, insulin resistance, type 2 diabetes mellitus, hyperlipidemia, and cardiovascular disease. It encompasses the entire spectrum of fatty liver diseases from simple steatosis to nonalcoholic steatohepatitis (NASH) with lobular/portal inflammation, hepatocellular necrosis, and fibrosis. Of those who develop NASH, 15-25\% will progress to end stage liver disease and hepatocellular carcinoma over 10-20 years. Its pathogenesis is complex, and involves a state of lipid accumulation due to increased uptake of free fatty acids into the liver, impaired fatty acid beta oxidation, and increased incidence of de novo lipogenesis. Plasma aminotransferases and liver ultrasound are helpful in the diagnosis of NAFLD/NASH, but a liver biopsy is often required for definitive diagnosis. Many new plasma biomarkers and imaging techniques are now available that should improve the ability to diagnose NAFLD noninvasively Due to its complexity and extrahepatic complications, treatment of NAFLD requires a multidisciplinary approach with excellent preventative care, management, and treatment. This review will evaluate our current understanding of NAFLD, with a focus on existing therapeutic approaches and potential pharmacological developments.
\end{abstract}

\section{Introduction}

Nonalcoholic fatty liver disease (NAFLD) represents a spectrum of disorders defined by the accumulation of fat in the liver. This spectrum ranges from simple steatosis to lobular inflammation with variable degrees of fibrosis leading to cirrhosis and even hepatocellular carcinoma (HCC) [1]. It is strongly associated with the metabolic syndrome and is the leading cause of chronic liver disease worldwide, with a prevalence of $15 \%-30 \%$ in Western populations $[2,3]$. The prevalence increases to $58 \%$ in overweight individuals and can be as high as $90 \%$ in obese individuals $[4,5]$. NAFLD was historically thought to be of little importance, but recent advances have shown that NAFLD can progress to nonalcoholic steatohepatitis (NASH) in up to $25 \%$ of patients $[1,6]$. NASH resembles alcoholic steatohepatitis (ASH) but occurs in individuals who do not consume excessive amounts of alcohol. Cirrhosis, which occurs in $25 \%$ of patients with $\mathrm{NASH}$, can result in liver failure, portal hypertension, and hepatocellular carcinoma [7]. However, the majority of deaths among individuals with NAFLD are attributed to cardiovascular disease and malignancy $[8,9]$. Unsurprisingly, these observations have fueled concern that NAFLD, either independently or in combination with other metabolic risk factors, could be an important risk factor for extrahepatic diseases. In this review, we will analyze the current theories for pathogenesis, accurate diagnosis, and management, including both current and novel treatments.

\section{Pathogenesis}

The initial theories for the pathogenesis of NAFLD were based on a "2-hit hypothesis"; however, this has now been expanded to a "3-hit hypothesis." The "1st hit" consisted of hepatic triglyceride accumulation that increases susceptibility of the liver to injury mediated by "2nd hit," such as proinflammatory cytokines (i.e., tumor necrosis factor 
TAble 1: Noninvasive diagnostic methods for nonalcoholic fatty liver disease.

\begin{tabular}{|c|c|c|}
\hline Method & Pros & Cons \\
\hline $\begin{array}{l}\text { Combined clinical and } \\
\text { plasma scoring systems }\end{array}$ & $\begin{array}{l}\text { Low cost, widely available, and acceptable } \\
\text { diagnostic accuracy }\end{array}$ & $\begin{array}{l}\text { Need for greater validation, decreased } \\
\text { accuracy for staging fibrosis }\end{array}$ \\
\hline Plasma CK-18 & $\begin{array}{l}\text { Can differentiate NAFLD or NASH and if } \\
\text { fibrosis present }\end{array}$ & $\begin{array}{l}\text { Not widely available, need for greater } \\
\text { validation }\end{array}$ \\
\hline Ultrasonography & Low cost, widely available, and safe & $\begin{array}{l}\text { Liver fat not quantifiable and low } \\
\text { sensitivity if }<30 \%\end{array}$ \\
\hline Transient elastography & $\begin{array}{l}\text { Safe, easy to use, and large probe for } \\
\text { obese patients available }\end{array}$ & $\begin{array}{l}\text { Expensive equipment, need for greater } \\
\text { validation }\end{array}$ \\
\hline $\begin{array}{l}\text { Magnetic resonance } \\
\text { elastography }\end{array}$ & $\begin{array}{l}\text { Quantifies liver fat and can evaluate for } \\
\text { fibrosis with good histology correlation }\end{array}$ & $\begin{array}{l}\text { Expensive equipment, expensive testing, } \\
\text { limited availability, and body size } \\
\text { constraints }\end{array}$ \\
\hline
\end{tabular}

alpha (TNF- $\alpha$ ) and interleukin-6 (IL-6)), adipokines, mitochondrial dysfunction, and oxidative stress, which in turn lead to steatohepatitis and/or fibrosis $[10,11]$. However, there is increasing recognition of the role that free fatty acids (FFA) play in directly promoting liver injury, leading to a modification of this theory. Universally, patients with NAFLD have insulin resistance (IR) which increases lipolysis from the adipose tissue [12]. In obesity and insulin resistance there is an increased delivery of FFA to the liver. These FFA either undergo lipid peroxidation or are esterified with glycerol to form triglycerides, leading to hepatic fat accumulation. There is now substantial evidence that FFA can directly cause toxicity by increasing oxidative stress and by activation of inflammatory pathways; therefore, hepatic triglyceride accumulation may be a protective mechanism by preventing the toxic effects of unesterified FFA $[13,14]$. In the healthy liver, cell death stimulates replication of mature hepatocytes, which replace the dead cells and reconstitute normal tissue function. However, in chronic liver injury, the development of fibrosis/cirrhosis is dependent on the efficacy of hepatocyte regeneration, and therefore cell death with impaired proliferation of hepatocyte progenitor cells represents the proposed " 3 rd hit" in NAFLD pathogenesis [15].

Diet is an important contributor to NAFLD mainly because excessive energy intake leads to obesity, which in turn increases the risk for NAFLD. However, not only the amount of energy but also the quality of the diet could play an important role in the development and progression of NAFLD. Diets rich in saturated fat and cholesterol and low in polyunsaturated fat and fiber are associated with IR and hepatic inflammation [16]. More recent evidence has linked intestinal microbiota to NAFLD pathogenesis. One study suggested an association between low percentage of fecal bacteroidetes and the presence of NASH, independent of diet and body mass index (BMI) [17]. Intestinal microbiota can be altered by the type of diet consumed and may contribute to NAFLD through several mechanisms, including salvaging energy from food, contributing to inflammation via cytokines by increasing intestinal permeability leading to endotoxemia, modulating the innate immune system, regulating bile acid, metabolizing dietary choline, and increasing endogenous ethanol by bacteria $[17,18]$.

\section{Staging of NAFLD}

NAFLD affects a substantial proportion of the population worldwide; however few patients who have the condition develop liver-related complications. Predicting which patients will develop progressive disease is problematic. To avoid the need for repeated liver biopsy to assess for NASH, recent advances have demonstrated newer noninvasive techniques that may be used to diagnose the amount of inflammation and fibrosis (see also Table 1).

3.1. Laboratory Variables. Cytokeratin (CK) 18 is the serum marker of NASH that has been the most validated marker to date. CK-18 fragments come from apoptosis of hepatocytes degraded by the enzyme caspase $3[19,20]$. CK-18 fragments can be investigated in liver tissue using immunostaining or measured in plasma using monoclonal antibodies. Studies suggest plasma CK-18 levels may help in distinguishing between simple steatosis from NASH, but the test is far from perfect with low overall sensitivity and specificity $[19,20]$.

To increase the accuracy of noninvasive markers of liver fibrosis, multiple markers and patient characteristics have been combined into mathematic models to produce predictive scores. The FibroTest is one algorithm, consisting of a combination of age, gender, bilirubin, g-glutamyltransferase, apolipoprotein A1, haptoglobin, and a2-macroglobulin. It has been validated in a variety of chronic liver diseases [21]. In studies, high scores have a very accurate predictive value for the presence of bridging fibrosis or cirrhosis but were unable to predict severity of liver fibrosis in one-third of patients with NAFLD [21]. Additional similar panels are available from different lab companies [22].

3.2. Imaging Assessment. Conventional ultrasound, CT, and MRI can noninvasively detect hepatic steatosis and have a sufficient level of accuracy in detecting cirrhosis with portal hypertension. However, they are far less reliable at detecting NASH and the associated stages of fibrosis. New imaging technologies, such as the ultrasonography-based transient elastography (FibroScan, Echosens, Paris, France) and magnetic resonance elastography (MRE), offer promise in determining severity of liver fibrosis associated with NASH. FibroScan, also known as transient elastography (TE), 
measures liver stiffness-a property originally validated for the hepatitis $C$ population-but is now being used in patients with NAFLD also [22-24]. TE sends a pulse through the skin, which is circulated through the liver. The velocity of the wave correlates with liver stiffness-the stiffer the liver the greater the degree of fibrosis. Yoneda et al. investigated the usefulness of the TE in NAFLD patients [22]. They found that there is a significant correlation between liver stiffness and fibrosis stage, which was confirmed by liver biopsy. Therefore, this measurement can be used in the NAFLD population to estimate the stage of fibrosis. Special consideration is needed for overweight and obese patients. Studies have shown that obesity (BMI $>30 \mathrm{~kg} / \mathrm{m}^{2}$ ) provides inaccurate LSMs. The use of a FibroScan XL probe has been shown to provide reliable liver stiffness measurement $[25,26]$ in this population.

In contrast to TE, MRE estimates the average degree of liver fibrosis throughout most of the liver parenchyma by assessing the propagation of mechanical waves through the tissue. First, shear waves are generated in the liver tissue by a driver attached to the abdominal wall. Magnetic resonance images are then obtained depicting the propagated shear waves, and finally, images of the shear waves are analyzed and used to generate quantitative maps of tissue stiffness, referred to as elastograms [27]. As the entire liver can be sequenced, the area of sampling is greatly increased, and the heterogeneous distribution of fibrosis is more commonly appreciated $[28,29]$ Studies thus far have shown that technical success rate of MRE was significantly higher than that of TE (94\% versus 84\%) [30]. Rather intriguing are the recent findings showing that MRE is useful in helping to identify patients with steatohepatitis, even prior to the onset of fibrosis [28]. In a retrospective study of 58 NAFLD patients, hepatic stiffness had high accuracy in discriminating patients with $\mathrm{NASH}$ from those with simple steatosis $($ AUROC $=0.93$, sensitivity $94 \%$, and specificity $73 \%$ by using a threshold of $2.74 \mathrm{kPa}$ ). NAFLD patients with inflammation (NASH) but no fibrosis have greater liver stiffness than those with simple steatosis and lower mean stiffness than those with fibrosis [28]. Despite these encouraging results, there are still issues of concern with MRE techniques. These include the increased acquisition time of scanning, the costs of the equipment, the expertise in analysis, body size limitations to fit in the scanner, and standardized thresholds of measurement.

The ultimate decision regarding whom and when to perform a liver biopsy should take into account whether the information likely to be obtained would affect the patient's care. There are 2 general indications for performing a liver biopsy in patients with suspected NAFLD: (1) confirming the diagnosis and staging the disease and (2) determining prognosis based on the severity of liver injury and fibrosis [31]. Liver biopsy is the only modality that can reliably distinguish between simple steatosis and NASH, as well as stage the extent of fibrosis. The prognosis of NAFLD depends on the severity of liver injury and fibrosis. While most studies suggest that there is no increased mortality associated with simple hepatic steatosis, mortality in patients with NASH, particularly those with advanced fibrosis and cirrhosis, is increased compared to the general population of same age and gender patients. The highest liver-related morbidity and mortality are undoubtedly among those patients with advanced (stage 3 or 4) fibrosis [32-34]. Additionally, the identification of early cirrhosis or advanced (bridging) fibrosis may alter management, as such patients should undergo upper endoscopy to screen for gastroesophageal varices and regular interval liver imaging to screen for hepatocellular carcinoma [34].

\section{Treatment and Management of NAFLD}

The goal of managing NAFLD is to improve steatosis and prevent fibrosis. No standard treatment currently exists; however, treating risk factors, such as obesity and IR, remains the focus of managing NAFLD.

4.1. Lifestyle Interventions. The primary approach to treat NAFLD involves elimination of the underlying risk factors. It has been shown that community-based lifestyle modification programs are effective in reducing and normalizing liver fat in NAFLD patients [35]. Maintaining a weight loss of 5-10\% significantly improves histological severity, but frequently occurring subsequent weight gain leads to the recurrence of NASH $[36,37]$. Even moderate physical exercise, such as treadmill walking, improves markers of apoptosis and insulin sensitivity in NAFLD [38]. The dietary composition is also of great importance. A $2 \%$ increment in energy intake from trans fats resulted in a $0.77 \mathrm{~cm}$ waist gain over 9 years, and reduction of harmful trans fats improved histological features in a mouse model despite persistent obesity $[39,40]$.

4.2. Bariatric Surgery. Lifestyle interventions, particularly weight loss, are often very difficult for patients to achieve and sustain. Bariatric surgery induces weight loss by reducing the size of a patient's stomach by either removing a portion of the stomach, using a gastric band, or by gastric bypass, and is considered in patients who have a BMI greater than $40 \mathrm{~kg} / \mathrm{m}^{2}$ or with a BMI of $35 \mathrm{~kg} / \mathrm{m}^{2}$ who have obesity-related comorbidities [41]. Small prospective studies have found that bariatric surgery improved insulin resistance and liver function tests and has demonstrated histological improvement in hepatic steatosis and fibrosis [42, 43]. However, a recent Cochrane review concluded that there is insufficient data due to a lack of well-designed randomized control study trials to determine if bariatric surgery is an effective treatment for NAFLD [44]. At this point, the usefulness of bariatric surgery as a treatment for NAFLD, particularly for inflammation and fibrosis, is not clear and patients need to be carefully selected to undergo the procedure.

4.3. Medical Treatment. Despite an increasing understanding of the mechanisms of NAFLD pathogenesis, there are few effective therapies available. Current treatments are primarily directed towards improving the metabolic factors which contribute to disease pathogenesis, such as reducing IR, improving diabetic control, and weight loss. The urgent need for specific treatments for NAFLD has made this a critical area of research, with particular focus on developing treatments which can reverse or prevent the more advanced stages of NASH. 
4.3.1. Current Therapies. Due to the key role IR plays in the pathogenesis of NAFLD, insulin sensitizing agents have played an important role in its management, with metformin and thiazolidinediones (TZD) being of particular interest. Metformin is used in the treatment of type 2 diabetes, as it lowers blood glucose and improves insulin sensitivity by decreasing hepatic gluconeogenesis, decreasing intestinal glucose absorption, and increasing fatty acid oxidation $[45,46]$. Clinical studies have investigated the use of metformin in the treatment of NAFLD, specifically looking at liver histology and aminotransferases. A pilot study to investigate the efficiency and safety of metformin in NAFLD patients by Nair et al. showed only a transient improvement in liver tests without a sustainable reduction in insulin sensitivity [47]. Additional studies have also failed to prove benefits of using metformin to improve liver histology [48, 49]. TZD are peroxisomal proliferator activated receptor- $\gamma$ (PPAR- $\gamma$ ) agonists that are used primarily in the type 2 diabetes population to help improve insulin sensitivity, promote hepatic fatty acid oxidation, and decrease hepatic lipogenesis [50, 51]. TZD use in NAFLD patients, specifically pioglitazone and rosiglitazone, has been shown to decrease hepatic fat and decrease cellular injury. Unfortunately, these medications have also been shown to cause weight gain, which is troublesome in an already obese patient [52]. Belfort et al. studied the effects of lifestyle modification and $45 \mathrm{mg}$ of pioglitazone per day on $55 \mathrm{NASH}$ patients with impaired glucose tolerance or type 2 diabetes [53]. The results indicated that diet and pioglitazone improved glycemic control and glucose tolerance, improved liver enzymes, and increased hepatic sensitivity. Conversely, another study found that discontinuing TZD therapy resulted in NASH recurrence, indicating that long-term use is necessary for successful treatment [54]. Overall, pioglitazone can be effective as a treatment for NASH; however, careful consideration is needed due to its possible side effects $[55,56]$.

Vitamin $\mathrm{E}$ is the main antioxidant that has emerged as a treatment for NASH with the hope that it can help improve oxidative stress in the liver. Several studies have been conducted to further analyze the benefits of administering high doses of vitamin E to NASH patients. One of the larger studies administered high doses of vitamin E (800 IU/day) in nondiabetic patients and found a reduction in hepatocellular inflammation, hepatic steatosis, and improvements in liver function tests. They concluded that vitamin $\mathrm{E}$ is an effective treatment for NASH patients without diabetes [56]. Unfortunately, a meta-analysis of over 100,000 people taking $400 \mathrm{IU} / \mathrm{d}$ of vitamin $\mathrm{E}$ found that there is an increase of all-cause mortality and an additional study found that its supplementation significantly increases the risk of developing prostate cancer $[57,58]$. Overall, caution needs to be taken when prescribing vitamin E, especially to males and diabetic NASH patients.

\subsubsection{Emerging Therapies}

Inhibitors of Inflammation. The severity of NASH and the risk of progression correlate with the level of hepatocyte injury which often includes apoptosis and its associated inflammation. Apoptosis involves cell death signaling pathways, which lead to the activation of caspases, cellular proteases that degrade structural proteins required for the cell survival [59]. Inhibition of caspases has been proposed as a therapeutic approach in inflammation-associated disease [60]. In a double-blind, randomized phase II study of 124 patients with NASH, GS-9450, an inhibitor of caspases 1, 8, and 9, reduced serum ALT and cytokeratin-18 fragments at 4 weeks of treatment [61]. However, the compound was later withdrawn due to safety concerns. Despite this, decreasing the inflammatory response associated with apoptosis in active $\mathrm{NASH}$ remains an attractive target to reduce fibrosis and prevent disease progression.

Glucagon-Like Peptide-1 (Glp-1). Glp-1 is an incretin which is released from enterocytes in response to nutrient uptake. Glp-1 regulates postprandial insulin release, inhibits glycolytic glucagon, and suppresses appetite [62]. While indirect and direct Glp-1 agonists have been introduced in the treatment of diabetes, their potential in NASH is less clear. Directacting Glp-1 agonists, exenatide and liraglutide, have shown some therapeutic efficacy in NASH. Liraglutide corrected impaired fatty acid beta-oxidation in a rodent model with high dietary trans fats and fructose-enriched drinking water [63]. In a high-fat model using wild-type C57Bl6 and ob/ob mice, an exenatide analogue attenuated weight gain and mitigated elevations of ALT and hepatic triglycerides [64]. Exenatide also reduces ER stress-related hepatocyte cell death and increased protective macroautophagy in response to treatment with saturated and unsaturated fatty acids [65]. Thus, enhancement of incretin signaling showed modest to considerable improvements in vitro and in animal models of NASH. Since these drugs have shown safety in patients with type 2 diabetes, clinical studies in patents with NAFLD are warranted.

Farnesoid X Receptor (FXR) Agonists. Bile acids (BAs), classically recognized as detergent-like compounds involved in lipid absorption and cholesterol homeostasis, have more recently been shown to modulate several metabolic pathways that regulate glucose, lipid, and energy homeostasis by targeting FXR [58-66]. In addition, FXRs mediate anti-inflammatory and antifibrotic properties, making them promising targets for the treatment of a number of metabolic and liver diseases $[67,68]$. FXR, a member of the nuclear receptor superfamily, is mainly expressed in liver, intestine, kidney, and, to a lesser extent, adipose tissue. It regulates a wide variety of target genes involved in the control of BAs synthesis and transport, lipid metabolism, and glucose homeostasis [69]. In particular, FXR controls glucose metabolism through regulation of hepatic gluconeogenesis and glycogenolysis, as well as regulation of peripheral insulin sensitivity in striated muscle and adipose tissue. Currently, obeticholic acid (OCA), a semisynthetic bile acid derivative, is being tested in patients with biopsy-proven NASH [70, 71]. From preliminary data, OCA appears to be a very promising possible treatment for NASH.

Probiotics. As discussed earlier in this review, the effect of a pr-inflammatory intestinal microbiome has been thought to 
be a contributor to the formation of NAFLD and NASH. In an open-label pilot study in 20 patients with biopsy-proven NASH, supplementation of a probiotic containing lactobacilli and bifidobacteria over a 6-month period improved hepatic steatosis, as determined by MRI and serum transaminases [72]. Together with the human randomized controlled study on fecal transplantation of a healthy microbiota in patients with insulin resistance, these recent reports support the role of microbiota in the pathogenesis of insulin resistance and $\mathrm{NASH}$, partly by reducing bacterial inflammatory triggers and nutrient modification [73]. It also hints to a role of prebiotics, which are nutrients that favor the growth of certain bacterial species, which may play a supportive role in the treatment of obesity and NASH [74]. Despite favorable results in these early studies, there is a need for larger longitudinal clinical trials to be able to determine the optimal dose, duration, and probiotic composition.

\section{Conclusion}

NAFLD is global challenge to our healthcare system and has put a large part of the world's population at risk of developing $\mathrm{NASH}$, cirrhosis, and liver cancer. Although we have an improved understanding of its epidemiology and pathogenesis, guidelines for treatment are changing as new information emerges. The development of noninvasive measures to assess inflammation and fibrosis are becoming more commonly used in practice but have not fully replaced the need for liver biopsy in many cases. Addressing the risk factors associated with NAFLD, such as IR, obesity and lipid levels remain the primary way to improve NAFLD; however, bariatric surgery, insulin sensitizing agents, and antioxidants may also be considered. Currently, pharmacological interventions aiming to improve insulin sensitivity without producing side effects, such as peripheral weight gain, and to reduce hepatocellular injury by reducing lipotoxicity are being developed.

\section{Conflict of Interests}

The authors declare that there is no conflict of interests regarding the publication of this paper.

\section{References}

[1] S. Caldwell and C. Argo, "The natural history of non-alcoholic fatty liver disease," Digestive Diseases, vol. 28, no. 1, pp. 162-168, 2010.

[2] G. Bedogni, L. Miglioli, F. Masutti, C. Tiribelli, G. Marchesini, and S. Bellentani, "Prevalence of and risk factors for nonalcoholic fatty liver disease: the dionysos nutrition and liver study," Hepatology, vol. 42, no. 1, pp. 44-52, 2005.

[3] C. P. de Oliveira, E. S. de Mello, V. A. F. Alves, S. M. Saviero, and E. Strauss, "Changes in histological criteria lead to different prevalences of nonalcoholic steatohepatitis in severe obesity," Annals of Hepatology, vol. 6, no. 4, pp. 255-261, 2007.

[4] C. Boza, A. Riquelme, L. Ibañez et al., "Predictors of nonalcoholic steatohepatitis (NASH) in obese patients undergoing gastric bypass," Obesity Surgery, vol. 15, no. 8, pp. 1148-1153, 2005.
[5] F. Harnois, S. Msika, J.-M. Sabaté et al., "Prevalence and predictive factors of non-alcoholic steatohepatitis (NASH) in morbidly obese patients undergoing bariatric surgery," Obesity Surgery, vol. 16, no. 2, pp. 183-188, 2006.

[6] K. J. P. Schwenger and J. P. Allard, "Clinical approaches to nonalcoholic fatty liver disease," World Journal of Gastroenterology, vol. 20, no. 7, pp. 1712-1723, 2014.

[7] C. A. Matteoni, Z. M. Younossi, T. Gramlich, N. Boparai, and A. J. McCullough, "Nonalcoholic fatty liver disease: a spectrum of clinical and pathological severity," Gastroenterology, vol. 116, no. 6, pp. 1413-1419, 1999.

[8] D. Kim, W. R. Kim, H. J. Kim, and T. M. Therneau, "Association between noninvasive fibrosis markers and mortality among adults with nonalcoholic fatty liver disease in the United States," Hepatology, vol. 57, no. 4, pp. 1357-1365, 2013.

[9] M. J. Armstrong, L. A. Adams, A. Canbay, and W.-K. Syn, "Extrahepatic complications of nonalcoholic fatty liver disease," Hepatology, vol. 59, no. 3, pp. 1174-1197, 2014.

[10] J. A. Marrero, R. J. Fontana, G. L. Su, H. S. Conjeevaram, D. M. Emick, and A. S. Lok, "NAFLD may be a common underlying liver disease in patients with hepatocellular carcinoma in the United States," Hepatology, vol. 36, no. 6, pp. 1349-1354, 2002.

[11] C. P. Day and O. F. W. James, "Steatohepatitis: a tale of two 'Hits'?" Gastroenterology, vol. 114, no. 4, pp. 842-845, 1998.

[12] E. Bugianesi, G. Marchesini, E. Gentilcore et al., "Fibrosis in genotype 3 chronic hepatitis $\mathrm{C}$ and nonalcoholic fatty liver disease: role of insulin resistance and hepatic steatosis," Hepatology, vol. 44, no. 6, pp. 1648-1655, 2006.

[13] A. E. Feldstein, N. W. Werneburg, A. Canbay et al., "Free fatty acids promote hepatic lipotoxicity by stimulating TNF- $\alpha$ expression via a lysosomal pathway," Hepatology, vol. 40, no. 1, pp. 185-194, 2004.

[14] K. Yamaguchi, L. Yang, S. McCall et al., "Inhibiting triglyceride synthesis improves hepatic steatosis but exacerbates liver damage and fibrosis in obese mice with nonalcoholic steatohepatitis," Hepatology, vol. 45, no. 6, pp. 1366-1374, 2007.

[15] J. Jou, S. S. Choi, and A. M. Diehl, "Mechanisms of disease progression in nonalcoholic fatty liver disease," Seminars in Liver Disease, vol. 28, no. 4, pp. 370-379, 2008.

[16] M. Mouzaki, E. M. Comelli, B. M. Arendt et al., "Intestinal microbiota in patients with nonalcoholic fatty liver disease," Hepatology, vol. 58, no. 1, pp. 120-127, 2013.

[17] M. D. Spencer, T. J. Hamp, R. W. Reid, L. M. Fischer, S. H. Zeisel, and A. A. Fodor, "Association between composition of the human gastrointestinal microbiome and development of fatty liver with choline deficiency," Gastroenterology, vol. 140, no. 3, pp. 976-986, 2011.

[18] J. K. Dowman, J. W. Tomlinson, and P. N. Newsome, "Systematic review: the diagnosis and staging of non-alcoholic fatty liver disease and non-alcoholic steatohepatitis," Alimentary Pharmacology \& Therapeutics, vol. 33, no. 5, pp. 525-540, 2011.

[19] A. E. Feldstein, A. Wieckowska, A. R. Lopez, Y.-C. Liu, N. N. Zein, and A. J. McCullough, "Cytokeratin-18 fragment levels as noninvasive biomarkers for nonalcoholic steatohepatitis: a multicenter validation study," Hepatology, vol. 50, no. 4, pp. 1072-1078, 2009.

[20] V. Ratziu, J. Massard, F. Charlotte et al., "Diagnostic value of biochemical markers (Fibro Test-FibroSURE) for the prediction of liver fibrosis in patients with non-alcoholic fatty liver disease," BMC Gastroenterology, vol. 6, article 6, 2006. 
[21] I. N. Guha, J. Parkes, P. Roderick et al., "Noninvasive markers of fibrosis in nonalcoholic fatty liver disease: validating the European liver fibrosis panel and exploring simple markers," Hepatology, vol. 47, no. 2, pp. 455-460, 2008.

[22] M. Yoneda, M. Yoneda, H. Mawatari et al., "Noninvasive assessment of liver fibrosis by measurement of stiffness in patients with nonalcoholic fatty liver disease (NAFLD)," Digestive and Liver Disease, vol. 40, no. 5, pp. 371-378, 2008.

[23] M. Ziol, A. Handra-Luca, A. Kettaneh et al., "Noninvasive assessment of liver fibrosis by measurement of stiffness in patients with chronic hepatitis C," Hepatology, vol. 41, no. 1, pp. 48-54, 2005.

[24] M. Yoneda, K. Fujita, M. Inamori, A. Nakajima, M. Tamano, and H. Hiraishi, "Transient elastography in patients with nonalcoholic fatty liver disease (NAFLD)," Gut, vol. 56, no. 9, pp. 1330-1331, 2007.

[25] R. P. Myers, G. Pomier-Layrargues, R. Kirsch et al., "Feasibility and diagnostic performance of the FibroScan XL probe for liver stiffness measurement in overweight and obese patients," Hepatology, vol. 55, no. 1, pp. 199-208, 2012.

[26] V. De Lédinghen, V. W.-S. Wong, J. Vergniol et al., “Diagnosis of liver fibrosis and cirrhosis using liver stiffness measurement: comparison between M and XL probe of FibroScan," Journal of Hepatology, vol. 56, no. 4, pp. 833-839, 2012.

[27] Y. K. Mariappan, K. J. Glaser, and R. L. Ehman, "Magnetic resonance elastography: a review," Clinical Anatomy, vol. 23, no. 5, pp. 497-511, 2010.

[28] J. Chen, J. A. Talwalkar, M. Yin, K. J. Glaser, S. O. Sanderson, and R. L. Ehman, "Early detection of nonalcoholic steatohepatitis in patients with nonalcoholic fatty liver disease by using MR elastography," Radiology, vol. 259, no. 3, pp. 749-756, 2011.

[29] M. Yin, J. A. Talwalkar, K. J. Glaser et al., "Assessment of hepatic fibrosis with magnetic resonance elastography," Clinical Gastroenterology and Hepatology, vol. 5, no. 10, pp. 1207-1213, 2007.

[30] L. Huwart, C. Sempoux, E. Vicaut et al., "Magnetic resonance elastography for the noninvasive staging of liver fibrosis," Gastroenterology, vol. 135, no. 1, pp. 32-40, 2008.

[31] G. A. Grandison and P. Angulo, "Can NASH be diagnosed, graded, and staged noninvasively?" Clinics in Liver Disease, vol. 16, no. 3, pp. 567-585, 2012.

[32] Z. M. Younossi, M. Stepanova, N. Rafiq et al., "Pathologic criteria for nonalcoholic steatohepatitis: interprotocol agreement and ability to predict liver-related mortality," Hepatology, vol. 53, no. 6, pp. 1874-1882, 2011.

[33] A. J. Sanyal, C. Banas, C. Sargeant et al., "Similarities and differences in outcomes of cirrhosis due to nonalcoholic steatohepatitis and hepatitis C," Hepatology, vol. 43, no. 4, pp. 682689, 2006.

[34] N. Bhala, P. Angulo, D. van der Poorten et al., "The natural history of nonalcoholic fatty liver disease with advanced fibrosis or cirrhosis: an international collaborative study," Hepatology, vol. 54, no. 4, pp. 1208-1216, 2011.

[35] V. W.-S. Wong, R. S.-M. Chan, G. L.-H. Wong et al., "Community-based lifestyle modification programme for nonalcoholic fatty liver disease: a randomized controlled trial," Journal of Hepatology, vol. 59, no. 3, pp. 536-542, 2013.

[36] E. Centis, R. Marzocchi, S. Di Domizio, M. F. Ciaravella, and G. Marchesini, "The effect of lifestyle changes in non-alcoholic fatty liver disease," Digestive Diseases, vol. 28, no. 1, pp. 267-273, 2010 .
[37] B. H. Goodpaster, J. P. DeLany, A. D. Otto et al., "Effects of diet and physical activity interventions on weight loss and cardiometabolic risk factors in severely obese adults: a randomized trial," The Journal of the American Medical Association, vol. 304, no. 16, pp. 1795-1802, 2010.

[38] C. E. Fealy, J. M. Haus, T. P. J. Solomon et al., "Short-term exercise reduces markers of hepatocyte apoptosis in nonalcoholic fatty liver disease," Journal of Applied Physiology, vol. 113, no. 1, pp. 1-6, 2012.

[39] P. Koh-Banerjee, N.-F. Chu, D. Spiegelman et al., "Prospective study of the association of changes in dietary intake, physical activity, alcohol consumption, and smoking with 9-y gain in waist circumference among 16587 US men," The American Journal of Clinical Nutrition, vol. 78, no. 4, pp. 719-727, 2003.

[40] B. A. Neuschwander-Tetri, D. A. Ford, S. Acharya et al., "Dietary trans-fatty acid induced NASH is normalized following loss of trans-fatty acids from hepatic lipid pools," Lipids, vol. 47, no. 10, pp. 941-950, 2012.

[41] H. P. Cotrim and C. Daltro, "Liver: does bariatric surgery reduce the severity of NAFLD?" Nature Reviews Gastroenterology and Hepatology, vol. 7, no. 1, pp. 11-13, 2010.

[42] A. R. Moschen, C. Molnar, A. M. Wolf et al., "Effects of weight loss induced by bariatric surgery on hepatic adipocytokine expression," Journal of Hepatology, vol. 51, no. 4, pp. 765-777, 2009.

[43] C. K. Furuya Jr., C. P. M. S. de Oliveira, E. S. de Mello et al., "Effects of bariatric surgery on nonalcoholic fatty liver disease: preliminary findings after 2 years," Journal of Gastroenterology and Hepatology, vol. 22, no. 4, pp. 510-514, 2007.

[44] N. C. Chavez-Tapia, F. I. Tellez-Avila, T. Barrientos-Gutierrez, N. Mendez-Sanchez, J. Lizardi-Cervera, and M. Uribe, "Bariatric surgery for non-alcoholic steatohepatitis in obese patients," Cochrane Database Systematic Reviews, vol. 1, Article ID CD007340, 2010.

[45] G. Zhou, R. Myers, Y. Li et al., "Role of AMP-activated protein kinase in mechanism of metformin action," Journal of Clinical Investigation, vol. 108, no. 8, pp. 1167-1174, 2001.

[46] A. Mazza, B. Fruci, G. A. Garinis, S. Giuliano, R. Malaguarnera, and A. Belfiore, "The role of metformin in the management of NAFLD," Experimental Diabetes Research, vol. 2012, Article ID 716404, 13 pages, 2012.

[47] S. Nair, A. M. Diehl, M. Wiseman, G. H. Farr Jr., and R. P. Perrillo, "Metformin in the treatment of non-alcoholic steatohepatitis: a pilot open label trial," Alimentary Pharmacology and Therapeutics, vol. 20, no. 1, pp. 23-28, 2004.

[48] V. Nobili, M. Manco, P. Ciampalini et al., "Metformin use in children with nonalcoholic fatty liver disease: an open-label, 24month, observational pilot study," Clinical Therapeutics, vol. 30, no. 6, pp. 1168-1176, 2008.

[49] J. W. Haukeland, Z. Konopski, H. B. Eggesbø et al., "Metformin in patients with non-alcoholic fatty liver disease: a randomized, controlled trial," Scandinavian Journal of Gastroenterology, vol. 44, no. 7, pp. 853-860, 2009.

[50] M. K. Oh, J. Winn, and F. Poordad, "Review article: diagnosis and treatment of non-alcoholic fatty liver disease," Alimentary Pharmacology \& Therapeutics, vol. 28, no. 5, pp. 503-522, 2008.

[51] L. B. van Wagner and M. E. Rinella, "The role of insulinsensitizing agents in the treatment of nonalcoholic steatohepatitis," Therapeutic Advances in Gastroenterology, vol. 4, no. 4, pp. 249-263, 2011. 
[52] S. H. Caldwell, C. K. Argo, and A. M. S. Al-Osaimi, “Therapy of NAFLD: insulin sensitizing agents," Journal of Clinical Gastroenterology, vol. 40, supplement 1, pp. S61-S66, 2006.

[53] R. Belfort, S. A. Harrison, K. Brown et al., "A placebo-controlled trial of pioglitazone in subjects with nonalcoholic steatohepatitis," The New England Journal of Medicine, vol. 355, no. 22, pp. 2297-2307, 2006.

[54] G. Lutchman, A. Modi, D. E. Kleiner et al., "The effects of discontinuing pioglitazone in patients with nonalcoholic steatohepatitis," Hepatology, vol. 46, no. 2, pp. 424-429, 2007.

[55] G. P. Aithal, J. A. Thomas, P. V. Kaye et al., "Randomized, placebo-controlled trial of pioglitazone in nondiabetic subjects with nonalcoholic steatohepatitis," Gastroenterology, vol. 135, no. 4, pp. 1176-1184, 2008.

[56] A. J. Sanyal, N. Chalasani, K. V. Kowdley et al., "Pioglitazone, vitamin E, or placebo for nonalcoholic steatohepatitis," The New England Journal of Medicine, vol. 362, no. 18, pp. 1675-1685, 2010.

[57] S. A. Harrison, S. Torgerson, P. Hayashi, J. Ward, and S. Schenker, "Vitamin E and vitamin C treatment improves fibrosis in patients with nonalcoholic steatohepatitis," American Journal of Gastroenterology, vol. 98, no. 11, pp. 2485-2490, 2003.

[58] E. R. Miller, R. Pastor-Barriuso, D. Dalal, R. A. Riemersma, L. J. Appel, and E. Guallar, "Meta-analysis: high-dosage vitamin E supplementation may increase all-cause mortality," Annals of Internal Medicine, vol. 142, no. 1, 2005.

[59] N. Alkhouri, C. Carter-Kent, and A. E. Feldstein, "Apoptosis in nonalcoholic fatty liver disease: diagnostic and therapeutic implications," Expert Review of Gastroenterology and Hepatology, vol. 5, no. 2, pp. 201-212, 2011.

[60] M. L. Shiffman, P. Pockros, J. G. McHutchison, E. R. Schiff, M. Morris, and G. Burgess, "Clinical trial: the efficacy and safety of oral PF-03491390, a pancaspase inhibitor-a randomized placebo-controlled study in patients with chronic hepatitis C," Alimentary Pharmacology \& Therapeutics, vol. 31, no. 9, pp. 969978, 2010

[61] V. Ratziu, M. Y. Sheikh, A. J. Sanyal et al., "A phase 2, randomized, double-blind, placebo-controlled study of GS9450 in subjects with nonalcoholic steatohepatitis," Hepatology, vol. 55, no. 2, pp. 419-428, 2012.

[62] M. Olaywi, T. Bhatia, S. Anand, and S. Singhal, "Novel antidiabetic agents in non-alcoholic fatty liver disease: a minireview," Hepatobiliary and Pancreatic Diseases International, vol. 12, no. 6, pp. 584-588, 2013.

[63] J. E. Mells, P. P. Fu, S. Sharma et al., "Glp-1 analog, liraglutide, ameliorates hepatic steatosis and cardiac hypertrophy in C57BL/6J mice fed a western diet," The American Journal of Physiology-Gastrointestinal and Liver Physiology, vol. 302, no. 2, pp. G225-G235, 2012.

[64] J. L. Trevaskis, P. S. Griffin, C. Wittmer et al., "Glucagon-like peptide-1 receptor agonism improves metabolic, biochemical, and histopathological indices of nonalcoholic steatohepatitis in mice," American Journal of Physiology: Gastrointestinal and Liver Physiology, vol. 302, no. 8, pp. G762-G772, 2012.

[65] S. Sharma, J. E. Mells, P. P. Fu, N. K. Saxena, and F. A. Anania, "GLP-1 analogs reduce hepatocyte steatosis and improve survival by enhancing the unfolded protein response and promoting macroautophagy," PLoS ONE, vol. 6, no. 9, Article ID e25269, 2011.

[66] A. F. Hofmann, "Bile acids: trying to understand their chemistry and biology with the hope of helping patients," Hepatology, vol. 49, no. 5, pp. 1403-1418, 2009.
[67] P. Lefebvre, B. Cariou, F. Lien, F. Kuipers, and B. Staels, "Role of bile acids and bile acid receptors in metabolic regulation," Physiological Reviews, vol. 89, no. 1, pp. 147-191, 2009.

[68] C. Thomas, R. Pellicciari, M. Pruzanski, J. Auwerx, and K. Schoonjans, "Targeting bile-acid signalling for metabolic diseases," Nature Reviews Drug Discovery, vol. 7, no. 8, pp. 678-693, 2008

[69] D. A. A. Hollman, A. Milona, K. J. van Erpecum, and S. W. C. van Mil, "Anti-inflammatory and metabolic actions of FXR: insights into molecular mechanisms," Biochimica et Biophysica Acta: Molecular and Cell Biology of Lipids, vol. 1821, no. 11, pp. 1443-1452, 2012.

[70] L. Adorini, M. Pruzanski, and D. Shapiro, "Farnesoid X receptor targeting to treat nonalcoholic steatohepatitis," Drug Discovery Today, vol. 17, no. 17-18, pp. 988-997, 2012.

[71] S. Mudaliar, R. R. Henry, A. J. Sanyal et al., "Efficacy and safety of the farnesoid $\mathrm{x}$ receptor agonist Obeticholic acid in patients with type 2 diabetes and nonalcoholic fatty liver disease," Gastroenterology, vol. 145, no. 3, pp. 574-582, 2013.

[72] V. W. Wong, G. L. Wong, A. M. Chim et al., "Treatment of nonalcoholic steatohepatitis with probiotics. A proof-ofconcept study," Annals of Hepatology, vol. 12, no. 2, pp. 256-262, 2013.

[73] A. Vrieze, E. van Nood, F. Holleman F et al., "Transfer of intestinal microbiota from lean donors increases insulin sensitivity in individuals with metabolic syndrome," Gastroenterology, vol. 143, no. 4, pp. 913.e7-916.e7, 2012.

[74] J. A. Parnell, M. Raman, K. P. Rioux, and R. A. Reimer, "The potential role of prebiotic fibre for treatment and management of non-alcoholic fatty liver disease and associated obesity and insulin resistance," Liver International, vol. 32, no. 5, pp. 701-711, 2012. 


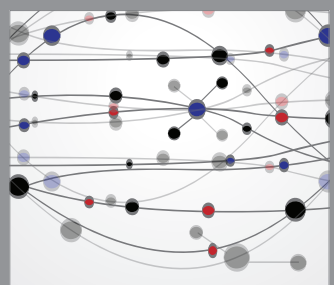

The Scientific World Journal
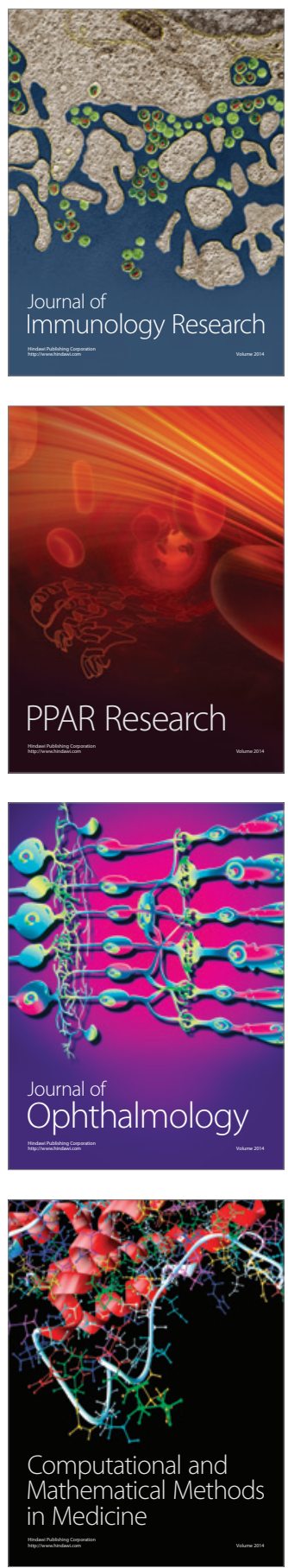

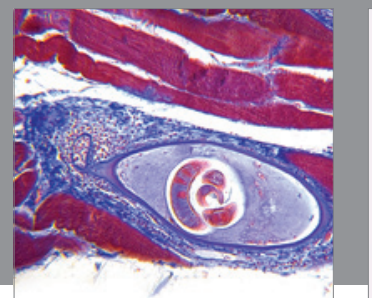

Gastroenterology

Research and Practice
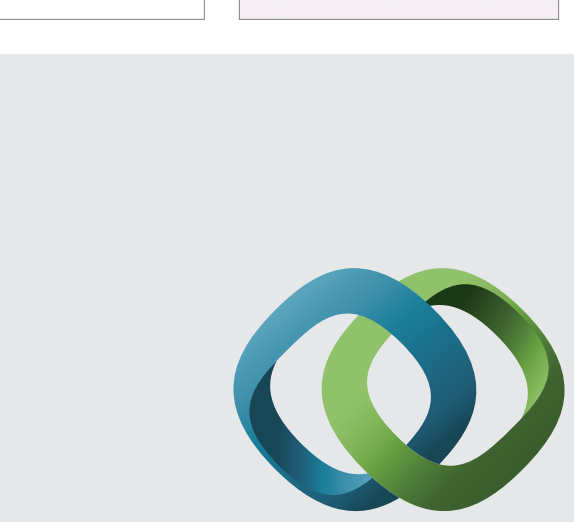

\section{Hindawi}

Submit your manuscripts at

http://www.hindawi.com
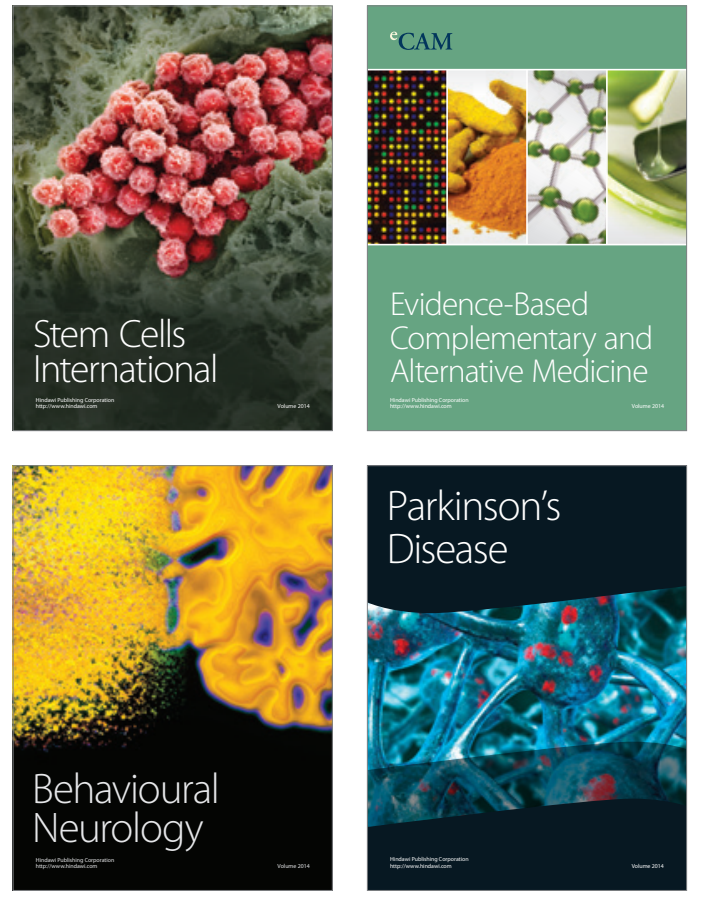
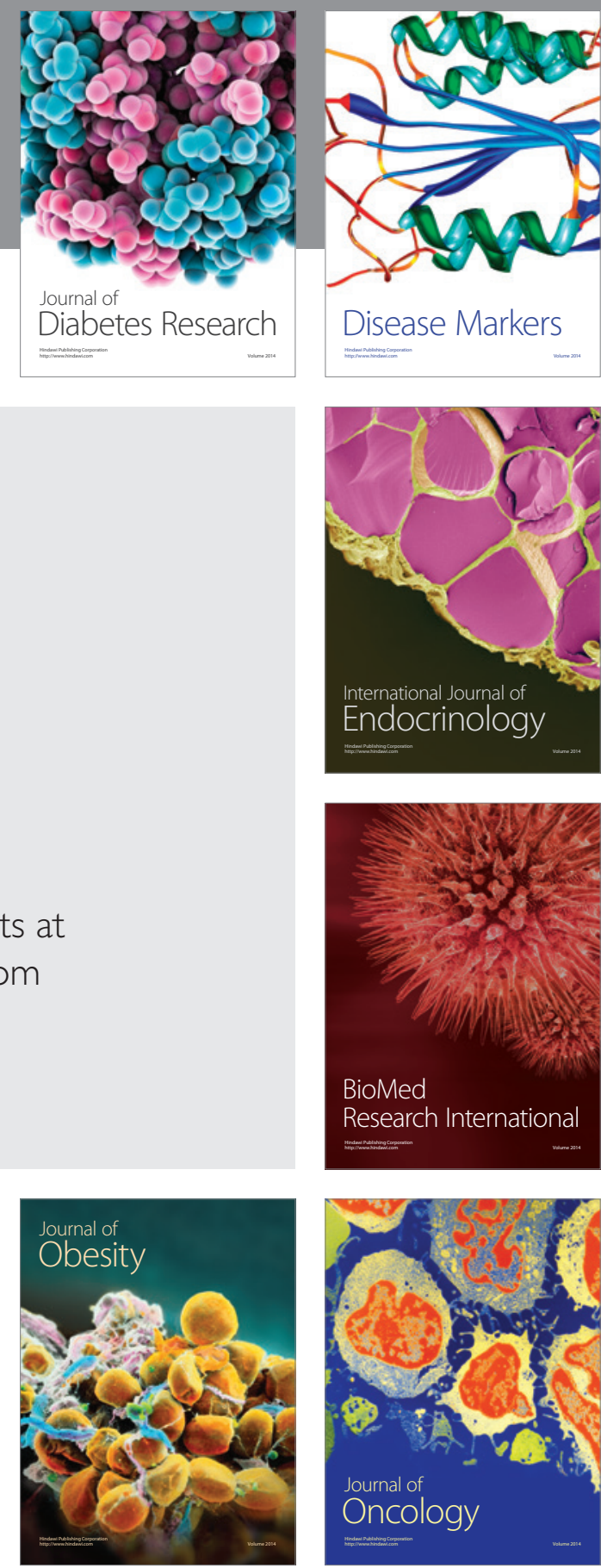

Disease Markers
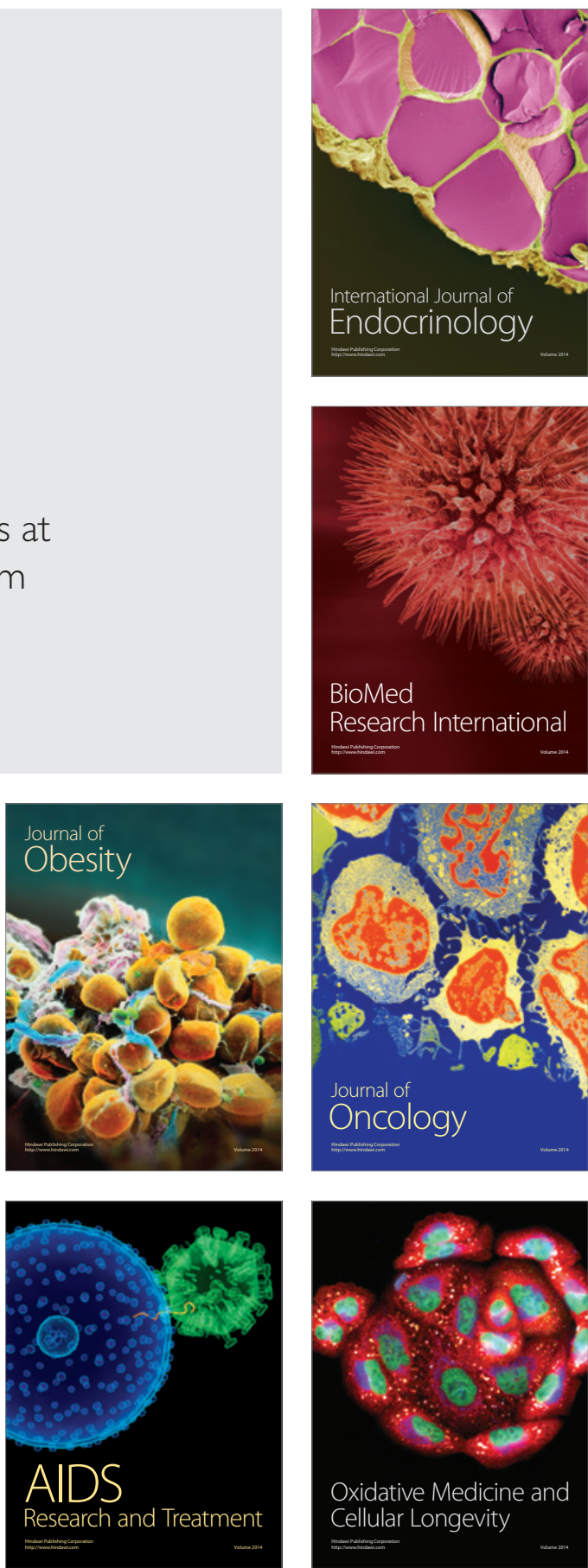